\title{
MULTI-WAVELENGTH EMISSION FROM THE FERMI BUBBLES. I. STOCHASTIC ACCELERATION FROM BACKGROUND PLASMA
}

\author{
K. S. Cheng ${ }^{1}$, D. O. Chernyshov ${ }^{1,2,3}$, V. A. Dogiel ${ }^{1,2,4}$, And C. M. Ko \\ ${ }^{1}$ Department of Physics, University of Hong Kong, Pokfulam Road, Hong Kong, China \\ 2 I.E. Tamm Theoretical Physics Division of P.N. Lebedev Institute of Physics, Leninskii pr. 53, 119991 Moscow, Russia \\ ${ }^{3}$ Institute of Astronomy, Department of Physics and Center for Complex Systems, National Central University, Jhongli, Taiwan \\ ${ }^{4}$ Moscow Institute of Physics and Technology (State University), 9, Institutsky lane, Dolgoprudny, 141707, Russia \\ Received 2013 December 17; accepted 2014 May 28; published 2014 June 27
}

\begin{abstract}
We analyze processes of electron acceleration in the Fermi bubbles in order to define parameters and restrictions of the models, which are suggested for the origin of these giant radio and gamma-ray structures. In the case of the leptonic origin of the nonthermal radiation from the bubbles, these electrons should be produced somehow in situ because of the relatively short lifetime of high-energy electrons, which lose their energy by synchrotron and inverse-Compton processes. It has been suggested that electrons in bubbles may be accelerated by shocks produced by tidal disruption of stars accreting onto the central black hole or a process of re-acceleration of electrons ejected by supernova remnants. These processes will be investigated in subsequent papers. In this paper, we focus on in situ stochastic (Fermi) acceleration by a hydromagnetic/supersonic turbulence, in which electrons can be directly accelerated from the background plasma. We showed that the acceleration from the background plasma is able to explain the observed fluxes of radio and gamma-ray emission from the bubbles, but the range of permitted parameters of the model is strongly restricted.
\end{abstract}

Key words: acceleration of particles - Galaxy: center - gamma rays: ISM

\section{INTRODUCTION}

Recent discovery of a mysterious, diffuse gamma-ray emission from the central portion of the Milky Way (Dobler et al. 2010; Su et al. 2010), which is seen as a giant feature (Fermi bubble) elongated perpendicular to the Galactic plane, was one of the marvelous discoveries in high-energy astrophysics. The gamma-ray spectrum from the bubbles is harder than elsewhere in the Galaxy, $d N / d E \propto E^{-2}$. This gamma-ray excess in the bubble region correlates with the earlier discovered socalled microwave haze observed by the Wilkinson Microwave Anisotropy Probe (WMAP) telescope as described by Finkbeiner (2004) and Dobler \& Finkbeiner (2008) and with the large-scale $\mathrm{X}$-ray emission region first evidenced by analyzing the ROSAT $1.5 \mathrm{keV}$ data, which clearly showed the characteristic of a bipolar flow (see Snowden et al. 1997). It was suggested that the ROSAT structure resulted from a fast wind that drove a shock into the halo gas with a velocity of $\sim 10^{8} \mathrm{~cm} \mathrm{~s}^{-1}$. This phenomenon requires an energy release $\sim 10^{55} \mathrm{erg}$ at the Galactic center (GC), which should be periodic in a timescale of $\sim 10^{7} \mathrm{yr}$ (Bland-Hawthorn \& Cohen 2003).

The WMAP haze was uncovered within the latitude range of about $30^{\circ}$ and was roughly bounded by the bipolar structure of X-rays. The existence of such haze implies a population of anomalously hard spectrum electrons toward the GC (Dobler 2012).

Recently, the Planck Collaboration (Ade et al. 2013) detected a residual diffuse emission in the range above the $30 \mathrm{GHz}$ region surroundings of the GC whose spatial distribution correlated nicely with the Fermi bubbles. At Galactic latitudes $|b|<30^{\circ}$, the microwave haze morphology is consistent with that of the Fermi gamma-ray bubbles (FBs). The correlation between these two features implies that the bubbles are real and their multi-wavelength emissions have a common origin. The derived spectrum is consistent with the power law favoring synchrotron radiation from electrons with a spectrum of $d N / d E \propto E^{-2.1}$.
This also implies a new mechanism for cosmic-ray acceleration in the center of our Galaxy. Further analysis of radio emission from the bubble region provided by Carretti et al. (2013) at $2.3 \mathrm{GHz}$ detected two giant, linearly polarized radio lobes emanating from the GC. The lobes extend $60^{\circ}$ and closely correspond to the Fermi bubbles. They concluded that the lobes are permeated by strong magnetic field strength up to $15 \mu \mathrm{G}$.

It is necessary to mention that the giant structures emanating from the center of our Galaxy are not unique. Even more giant structures are clearly seen in the direction of Cen-A in GHz radio (Junkes et al. 1993; Feain et al. 2011), GeV (Yang et al. 2012), and $\mathrm{TeV}$ (Aharonian et al. 2009) gamma-ray ranges. Recently, Stawarz et al. (2013) found X-ray features in the lobe of Cen-A, which they interpreted as emission of relativistic electrons in situ accelerated in the Cen-A lobes up to energies $\sim 10 \mathrm{TeV}$. Giant $\mathrm{X}$-ray and radio lobes (bubbles) were found also in NGC 3801 (Croston et al. 2007), Mrk 6 (Mingo et. al 2011), and Circinus Galaxy (Mingo et. al 2012).

The origin of the bubbles is actively discussed in the literature. These models include some phenomenological assumptions about processes of energy release and particle production in the bubbles. Thus, the assumed energy release in the GC needed for the bubble formation ranges from $10^{40} \mathrm{erg} \mathrm{s}^{-1}$, supplied by star formation regions as assumed by Crocker \& Aharonian (2011), to a hypothetical scenario of a single accretion with the released energy of about $10^{56}$ erg (see, e.g., Guo et al. 2012; Zubovas \& Nayakshin 2012) when a massive molecular clouds or a star cluster was captured by the central black hole ten million years ago.

Different mechanisms of gamma-ray production in the bubbles are suggested to explain the observed flux from the bubbles. Crocker \& Aharonian (2011) and Zubovas \& Nayakshin (2012) suggested that gamma-ray emission from the bubbles have hadronic origin, i.e., gamma-ray photons are produced by collisions of relativistic protons with that of the background gas. Alternatively, these gamma-rays can be produced by the 
inverse-Compton scattering of relativistic electrons on background photons (leptonic model), and the same electrons generate radio and microwave emission from the bubbles via synchrotron (see, e.g., Su et al. 2010). There may be several sources (processes) which generate relativistic electrons in the bubbles.

1. In situ stochastic acceleration by MHD turbulence near the bubble surface (Mertsch \& Sarkar 2011).

2. Acceleration by shocks originated from repeated tidal disruption of stars captured by the SMBH at the GC (Cheng et al. 2011).

3. Acceleration within jets near the GC about $\sim 10^{6} \mathrm{yr}$ ago, and subsequent electron transfer into the bubble by convective flows (Guo et al. 2012; Yang et al. 2013).

The goal of theoretical models is to explain a number of emission parameters that have several remarkable features in the bubbles (see Su et al. 2010; Dobler 2012; Ade et al. 2013).

1. The structures are symmetrically elongated in the direction perpendicular to the Galactic plane.

2. Spectra of radio emission from the bubbles are harder than anywhere in the Galaxy, and the assumed spectrum of electrons is a power law $\propto E^{-2}$.

3. The spatial distribution of emission in the bubbles shows sharp edges of the bubbles.

4. The surface emissivity is almost uniform inside the bubbles, although findings of Hooper \& Slatyer (2013) might indicate that some features of the gamma-ray spectrum at latitudes $|b| \leqslant 20^{\circ}$ could be interpreted as a contribution from the dark matter annihilation near the GC.

Almost all FB models on the spectra of nonthermal emission depend on free parameters that allow the data derived from observations to be more or less easily reproduced. We deem that these parameters can be restricted quantitatively if we estimate how many high-energy particles can be generated by this or that mechanism of acceleration. The number of high-energy particles has not previously been estimated, although this parameter gives a strong restriction on acceleration processes as we intend to show in our investigations.

Below, we analyze leptonic models of gamma-rays from the FB in which gamma-rays are generated by inverse Compton. Here, we do not suggest a new model of acceleration; instead, our goal is to understand whether the existing models are able to provide enough emitting particles. We analyze this aspect of stochastic acceleration and shock acceleration models in this and subsequent papers.

The stochastic acceleration can be provided by interaction of charged particles with a hydromagnetic turbulence that is excited in the halo by jets (see, e.g., Zubovas \& Nayakshin 2012) or by a shock (as assumed by Mertsch \& Sarkar 2011). Alternatively, this acceleration is provided by the interaction of particles with a supersonic turbulence (shocks) that arises from tidal disruptions of stars captured by the SMBH at the GC as proposed by Cheng et al. (2012). To provide seeds for stochastic acceleration in the bubbles, there are no other evident sources of electrons except those from the background plasma or those injected from the Galactic plane by sources such as supernova remnants (SNRs), pulsars, jets, etc., or from $p-p$ collisions in the halo (secondary electrons). We aim to define model restrictions for different processes of particle acceleration in the bubbles. In particular, the goal of our analysis is to define whether the processes of stochastic or multiple shock acceleration are able to accelerate electrons up to high energies and at what conditions they provide relativistic electrons in the Bubble with the required density and spectrum.

We start from the case of stochastic acceleration from a background plasma that has it own specificity. Particularly, in order to estimate the number of accelerated electrons, we should estimate a flux of thermal electrons running away into the region of acceleration which is generated by Coulomb collisions of thermal particles (see for details Gurevich 1960; Dogiel 2000). Therefore, we included into the kinetic equation two additional terms describing the Coulomb scattering. The kinetic equations with terms describing particle injection from a thermal plasma have not been investigated in previous models of the FBs.

\section{IN SITU ACCELERATION FROM A BACKGROUND PLASMA-GENERAL REMARKS.}

The kinetic equation for the distribution function of electrons, $f(p, t)$, in the case of in situ acceleration has the form

$$
\frac{\partial f}{\partial t}+\frac{1}{p^{2}} \frac{\partial}{\partial p} p^{2}\left[\left(\frac{d p}{d t}\right)_{C} f-\left\{D_{C}(p)+D_{F}(p)\right\} \frac{\partial f}{\partial p}\right]+\frac{f}{\tau}=0,
$$

where the dimensionless momentum $p$ is in units of $m c$. The distribution function $f$ includes the thermal and nonthermal components of the particle distribution. The coefficient $(d p / d t)_{C}$ describes particle ionization/Coulomb energy losses, and $D_{C}(p)$ describes diffusion in the momentum space due to Coulomb collisions (for equations for these term, see Landau \& Lifshitz 1981). The parameter $\tau$ is the lifetime of particles in the region of acceleration, e.g., due to escape from there. The stochastic (Fermi) acceleration is described as diffusion in the momentum space with the coefficient $D_{F}(p)$, whose value is determined by the frequency of particle collisions with, e.g., magnetohydrodynamic fluctuations or shocks. In the case of scattering by resonant MHD waves, the coefficient has the form (see, e.g., Berezinskii et al. 1990)

$$
D_{F}(p)=2 p^{2}\left(\frac{v_{a}}{v}\right)^{2} \int_{0}^{1} d \mu\left(1-\mu^{2}\right) \frac{v_{\mu}^{+} v_{\mu}^{-}}{\left(v_{\mu}^{+}+v_{\mu}^{-}\right)}
$$

where

$$
\nu_{\mu}^{ \pm} \simeq 2 \pi^{2}\left|\omega_{H}\right| \frac{k_{\mathrm{res}} W^{ \pm}\left(k_{\mathrm{res}}\right)}{H^{2}} .
$$

$H$ is a strength of the large-scale magnetic field, and $W^{ \pm}$is the power spectrum of MHD waves propagating along a magnetic field line in the both directions. Here, $\mu$ is the cosine of particle pitch angle and

$$
k_{\mathrm{res}}=\left|\frac{e H}{p c^{2} m \mu}\right|, \quad \omega_{H}=\frac{e H}{m_{e} c},
$$

where $p$ is in units of $m c$.

In the case of stochastic acceleration of electrons by a supersonic turbulence, the coefficient of momentum diffusion is (see Bykov \& Toptygin 1993)

$$
D_{F}(p) \sim \frac{u^{2}}{c l_{\mathrm{sh}}} p^{2}
$$

where $u$ is the shock velocity and $l_{\mathrm{sh}}$ is the average separation between two shocks. The acceleration by a supersonic turbulence is possible if the mean path length of electrons determined by energy losses and spatial diffusion in the intershock medium is larger than the separation between shocks. 
The acceleration is effective when the rate of acceleration exceeds that of losses, i.e., for $p>p_{\text {inj }}$,

$$
p_{\text {inj }} \sim D_{F}(p) /(d p / d t)_{C}
$$

A naive assumption could be that in the range $p<p_{\text {inj }}$, the spectrum is Maxwellian, and for $p>p_{\text {inj }}$, the spectrum is nonthermal (power law). However, calculations of the nonthermal component is nontrivial. As Gurevich (1960) showed, the acceleration distorted the equilibrium Maxwellian spectrum of background particles because of the flux of particles running away into the acceleration region. Even in the case when only a small part of thermal particles are accelerated and the coefficient of the kinetic equation $\left((d p / d t)_{C}\right.$ and $\left.D_{C}\right)$ are determined by the Maxwellian part of the spectrum, that makes Equation (1) linear; a very broad transfer region between the thermal and nonthermal parts of the spectrum is generated by the acceleration. The calculation showed that the number of accelerated particles was larger than if followed from trivial estimates. This linear approximation was used by Dogiel (2000) and Dogiel (2007), who interpreted nonthermal X-ray emission of galaxy clusters by in situ accelerated electrons.

However, approximation of Gurevich (1960) does not take into account a backward reaction of accelerated particles on the thermal component. This effect can be analyzed if the coefficients of Equation (1), i.e., $(d p / d t)_{C}$ and $D_{C}$, are calculated for the total distribution function which includes both thermal and nonthermal components (for details, see Landau \& Lifshitz 1981). Analysis of the nonlinear version of Equation (1) was provided by Wolfe \& Melia (2006) and Petrosian \& East (2008), who showed that the energy supplied by sources of stochastic acceleration was quickly absorbed by the thermal plasma because of the ionization/Coulomb energy losses of accelerated particles. As a result, this acceleration is accompanied mainly by plasma overheating while a tail of nonthermal particles is not formed, i.e., the effect of stochastic acceleration is negligible.

This conclusion was later revised by Chernyshov et al. (2012), who derived from analytical and numerical calculations that the efficiency of stochastic acceleration depended strongly on parameters of acceleration. For some conditions, the conclusion of Wolfe \& Melia (2006) and Petrosian \& East (2008) holds, i.e., plasma overheating does occur. However, there are conditions under which the acceleration forms a prominent nonthermal tail while the background plasma is not overheated.

Wolfe \& Melia (2006) and Chernyshov et al. (2012) presented the coefficient $D_{F}(p)$ in an arbitrary form as

$$
D_{F}(p)=\alpha p^{\varsigma} \theta\left(p-p_{0}\right)
$$

where $\alpha, \varsigma$, and $p_{0}$ are arbitrary parameters, i.e., the acceleration is effective in the momentum range $p>p_{0}$. We notice, however, that there are physical reasons for a cutoff at $p_{0}$, e.g., it may occur in the MHD spectrum of turbulence due to processes of wave absorption by accelerated cosmic rays (CRs). In the Appendix, we presented a qualitative estimation for $p_{0}$. We showed that for an appropriate combination of parameters, the value of $p_{0}$ is about 0.2 . However, this analysis is given as an illustration of potential possibility for a cutoff in a low momentum range and cannot be considered for comparison with the $p_{0}$ values derived in Section 3 from numerical simulations.

Here, we outline the analysis of in situ particle acceleration by stochastic acceleration in the Fermi bubbles. For details, we refer the reader to Chernyshov et al. (2012).
The total power supplied by external sources to electrons is determined from Equation (1) by the following integral:

$$
\dot{W}=-V_{F B} \int_{p_{0}}^{p_{\max }} \mathcal{E} \frac{\partial}{\partial p}\left[p^{2} D_{F} \frac{\partial f}{\partial p}\right] d p
$$

where $\mathcal{E}$ is the particle kinetic energy and $V_{F B} \sim 10^{67} \mathrm{~cm}^{3}$ is the volume of the bubble.

For simplicity, we present equations from Chernyshov et al. (2012) for large enough $p_{0}$. The flux of particles, $S$, running away to the acceleration region can be presented as

$$
S=\alpha(\varsigma+1) p_{0}^{\varsigma+1} \sqrt{\frac{2}{\pi}} \frac{N}{T} \exp \left(-\frac{\mathcal{E}_{0}}{T}\right)\left[1+\frac{\alpha(\varsigma+1) p_{0}^{\varsigma+1}}{A\left(p_{0}^{2}+1\right)}\right]
$$

where $N$ and $T$ are the density and the temperature of background plasma,

$$
\mathcal{E}_{0}=\sqrt{p_{0}^{2}+1}-1
$$

and

$$
A=4 \pi r_{e}^{2} c N \ln \Lambda .
$$

Here, $r_{e}$ is the electron radius, and $\ln \Lambda$ is the Coulomb logarithm. The spectrum of nonthermal particles can be presented as

$$
f(p)=\bar{f}(\bar{p} / p)^{\varsigma+1},
$$

where $\bar{f}$ is determined from the conditions by fitting between the thermal and nonthermal components of the spectrum, and $\bar{p}$ is the momentum value at this boundary.

In the quasi-stationary approximation when variations of $f$ are quite small, the nonthermal component can be presented as

$$
f(p)=-\frac{S}{\alpha(\varsigma+1)} p^{-\varsigma-1} \text { for } \quad p>p_{0} .
$$

The temperature variations due to the runaway flux $S$ and the Coulomb losses of nonthermal particles can be presented in the simplest case as (for a more accurate estimate, see Chernyshov et al. 2012)

$$
\frac{d T}{d t}=\frac{2 S}{3 N}\left[\frac{A Q\left(p_{0}, \varsigma\right)}{\alpha(\varsigma+1)}-\mathcal{E}_{0}\right]
$$

where

$$
Q\left(p_{0}, \varsigma\right)=\int_{p_{0}}^{\infty} x^{-\varsigma} \sqrt{x^{2}+1} d x
$$

\section{PARAMETERS OF THE MODEL OF IN SITU STOCHASTIC ACCELERATION IN THE FERMI BUBBLES}

Parameters of plasma in the FB are not well known. Below, for calculations, we accept them as they presented in $\mathrm{Su}$ et al. (2010), namely, the density $N=10^{-2} \mathrm{~cm}^{-3}$ and the temperature $T=2 \mathrm{keV}$. Estimations of the magnetic field strength in the FB ranges from several $\mu \mathrm{G}$ up to $15 \mu \mathrm{G}$ (see Strong et al. 2010; Jones et al. 2012; Carretti et al. 2013). The goal of these calculations is to reproduce the following characteristics of nonthermal emission from the FB.

1. The observed spectrum of gamma-rays has a cutoff at the energy of about $100 \mathrm{GeV}$ (Su et al. 2010), which corresponds to the maximum electron energy of about 0.3 TeV (see, e.g., Cheng et al. 2011). 
2. The total gamma-ray flux at energies $E>1 \mathrm{GeV}$ is $F_{\gamma} \simeq$ $4 \times 10^{37} \mathrm{erg} \mathrm{s}^{-1}$, and the spectrum can be approximated by $E_{\gamma}^{-2}$ in the range $1-100 \mathrm{GeV}$ (Su et al. 2010). This condition restricts the number of accelerated electrons.

3 . The radio flux from the bubble in the frequency range $20-60 \mathrm{GHz}$ is $(1-5) \times 10^{36} \mathrm{erg} \mathrm{s}^{-1}$, and the spectral index of radio emission is about -0.51 (see Finkbeiner 2004; Jones et al. 2012; Ade et al. 2013).

4. The power of potential sources of energy release in the GC cannot exceed the value of about $10^{40}$ for star formation regions (Crocker \& Aharonian 2011) and $10^{41} \mathrm{erg} \mathrm{s}^{-1}$ for tidal processes (Cheng et al. 2011).

5. The mechanism of particle acceleration should effectively generate nonthermal particles and not overheat the plasma (Chernyshov et al. 2012).

From these conditions, a necessary set of acceleration parameters, $\alpha, \varsigma, \tau$, and $p_{0}$, can be estimated using numerical simulations.

Assuming that the gamma-ray emission is produced by the accelerated relativistic electrons via inverse-Compton scattering, we calculated the intensity of gamma-ray emission along the line of sight $\mathbf{l}$ from the integral

$$
\begin{aligned}
I_{\gamma}\left(t, E_{\gamma}, \mathbf{l}\right)= & \frac{1}{4 \pi} \int_{\mathbf{l}} d \mathbf{l} \int_{\epsilon} n(\epsilon, \mathbf{r}) d \epsilon \int_{p} p^{2} f(\mathbf{r}, p, t) \\
& \times\left(\frac{d^{2} \sigma}{d \epsilon d p}\right)_{K N} d p .
\end{aligned}
$$

Here, $n(\epsilon, r)$ is the spatial distribution of background photons with the energy $\epsilon$ which was taken from Ackermann et al. (2012); $\left(d^{2} \sigma / d \epsilon d p\right)_{K N}$ is the Klein-Nishina cross-section taken from Blumenthal \& Gould (1970).

The cutoff in the electron spectrum can be derived from the balance between the acceleration and the energy losses

$$
p_{c}=\left(\frac{\alpha(\varsigma+1)}{\beta}\right)^{\frac{1}{3-\varsigma}}
$$

if $\varsigma<3$. Here, the synchrotron and inverse-Compton losses are presented as $d p / d t=\beta p^{2}$. For estimates, we took $H=5 \mu \mathrm{G}$, the density of optical photons $w_{o p}=1.6 \mathrm{eV} \mathrm{cm}^{-3}$, and the density of IR photons $w_{\mathrm{IR}}=0.33 \mathrm{eV} \mathrm{cm}^{-3}$ (see Ackermann et al. 2012; Carretti et al. 2013), which gives $\beta=1.5 \times 10^{-19} \mathrm{~s}^{-1}$.

As follows from calculations of Cheng et al. (2011), the electrons should be accelerated in the FB up to $E_{\max } \simeq 0.3 \mathrm{TeV}$ (condition 1). Then, from Equation (17), we can derive the function $\alpha(\varsigma)$.

In the case of stochastic acceleration, the coefficients of momentum, $D_{F}(p)$, and spatial, $K(p)$, diffusion are proportional to each other (see, e.g., Berezinskii et al. 1990):

$$
K(p) D_{F}(p) \approx \frac{p^{2} v^{2}}{6},
$$

where $v$ is the characteristic velocity of turbulence. Then, the escape time $\tau$ in Equation (1) is

$$
\tau \approx \frac{L^{2}}{4 K} \approx \frac{3 L^{2} D_{F}}{2 p^{2} v}=\frac{3 \alpha L^{2}}{2 v^{2}} p^{\varsigma-2},
$$

where $L<3 \mathrm{kpc}$ is the size of acceleration region.

The effect of escape is the steepening of the spectra of acceleration particles in comparison with the approximation (12). Then,

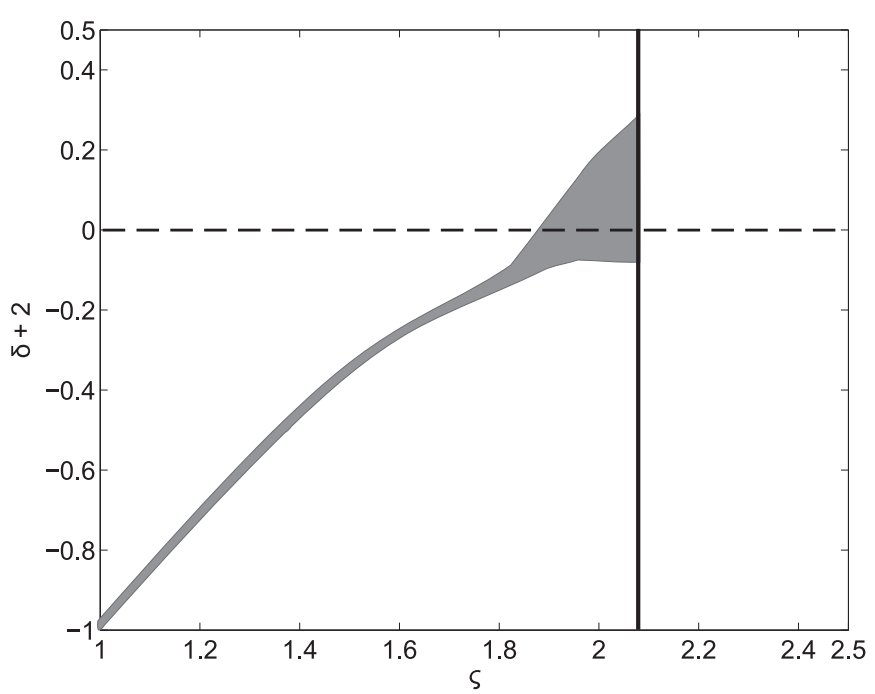

Figure 1. Function $\delta(\varsigma)$ derived from conditions 2 and 3 .

for the known function $\alpha(\varsigma)$, we can derive from Equation (1) the escape time $\tau(\alpha)$, and thus the spectrum of electrons that generates the radio flux from the FB as $I_{r} \propto v^{-0.5}$ (condition 3).

From Equation (16), we numerically can find the value of $\varsigma$ at which the spectrum of gamma-rays is power law $\left(I_{\gamma} \propto E_{\gamma}^{\delta}\right)$ with the spectral index of gamma-rays $\delta \simeq-2$ (condition 2 ). Variations of $\delta(\varsigma)$ calculated numerically are shown in Figure 1. As one can see from this figure, the required value of $\delta$ is obtained if $\varsigma \simeq 2$. For other values of $\varsigma$, the solution of (1) does not reproduce the observed gamma-ray spectrum from the FB. At that, the necessary value of $\tau$ is about $1.1 \times 10^{13} \mathrm{~s}$ and $\alpha \simeq 1.6 \times 10^{-14} \mathrm{~s}^{-1}$.

The next step of our calculations is to define whether the acceleration with the derived parameters $\alpha, \varsigma$, and $\tau$ can provide the necessary number of relativistic electrons to reproduce the observed intensity of the radio and gamma-ray emission from the FB. As one can see from Equations (9) and (13), the number of accelerated electrons depends on the cutoff momentum $p_{0}$, the larger $p_{0}$, and the smaller number of accelerated particles. However, for the value of $\alpha$ fixed from the cutoff position in the observed FB gamma-ray flux, the maximum value of $\zeta$ is determined by the density of electrons needed for the observed gamma-ray flux from the FBs and, in this respect, is independent of other parameters of the model. Just because of this effect, the parameter $\varsigma$ cannot be larger than 2.1 , as shown by the vertical line in Figure 1. We also note that although $\varsigma$ is a function of $\alpha$, its estimates from the electron spectrum (see Equation (13)) or from the cutoff position (see Equation (17)) depend logarithmically on $\alpha$, and, roughly, this dependence is neglected in calculations presented in Figure 1.

In Figure 2 (left panel), we showed the cutoff momentum $p_{0}(T)$ at which the number of accelerated electrons is high enough for the observed intensity of gamma-ray emission from the FB (condition 2). The numerical calculations were performed for the two values of the plasma density in the FB: the plasma densities $N=10^{-2} \mathrm{~cm}^{-3}$ (dashed line) and $N=3 \times 10^{-3} \mathrm{~cm}^{-3}$ (solid line). One can see that the acceleration can provide enough accelerated electrons if the temperature of background plasma is higher than $\sim 1 \mathrm{keV}$.

On the other hand, as was shown by Chernyshov et al. (2012), the value of $p_{0}$ should not be too small; otherwise, the plasma is overheated by the acceleration particles (condition 5). 

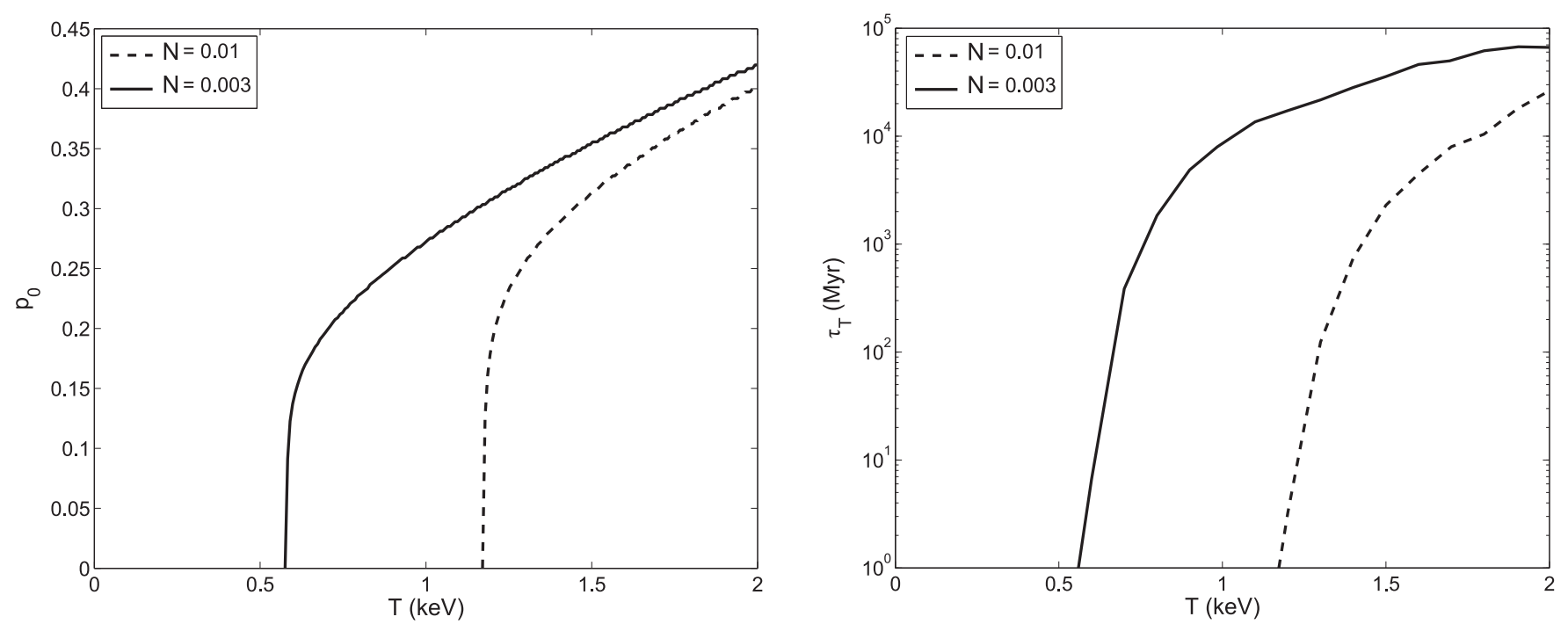

Figure 2. (Left panel) Function $p_{0}(T)$ derived from the condition that the number of accelerated electrons is enough to reproduce the observed the nonthermal emission from the FB. (Right panel) Time of plasma heating $\tau_{T}(T)$ derived from Equation (21).

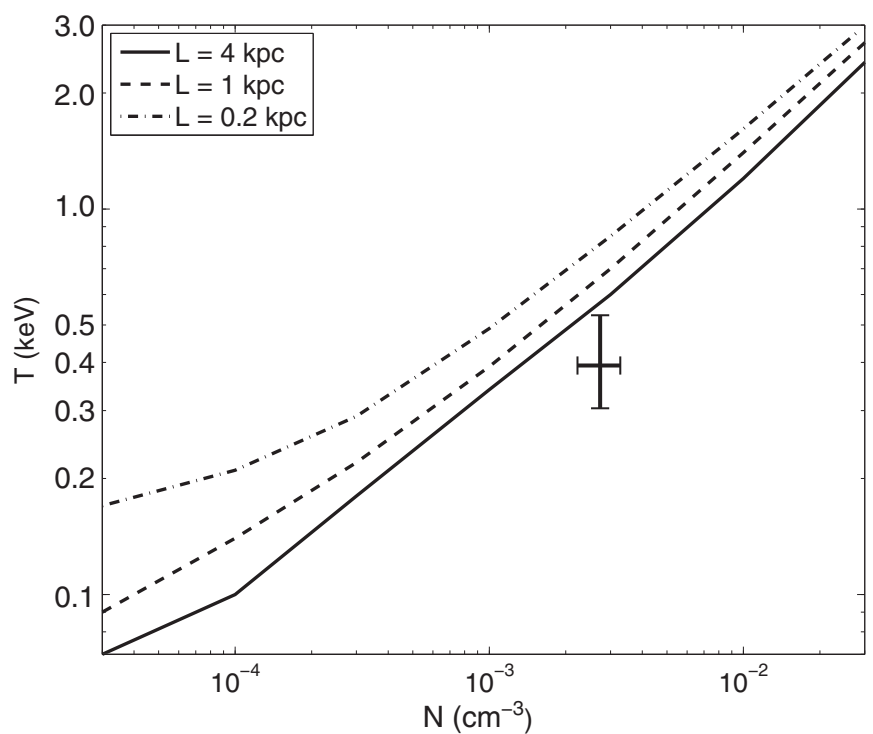

Figure 3. Minimum plasma temperature $T$ required for acceleration for a given density of background plasma $N$. The cross marks the observations of the Fermi bubbles by Suzaku (Kataoka et al. 2013).

This condition can be presented as an inequality,

$$
\tau_{\mathrm{acc}}<\tau_{T},
$$

where the acceleration time $\tau_{\text {acc }} \sim 1 / \alpha$ and the characteristic time of temperature variation $\tau_{T}$ is

$$
\tau_{T}=\frac{T}{d T / d t},
$$

where temperature variations due to heating by the accelerated electrons are described by Equation (14).

From the derived dependence $p_{0}(T)$, we calculated from Equation (21) the time of plasma heating $\tau_{T}$. The results are shown in Figure 2 (right panel). The derived acceleration time is about $\tau_{\text {acc }} \simeq 2$ Myr. Then, the acceleration is possible if $\tau_{\text {acc }}<\tau_{T}$. As is clear from the figure, this condition is realized for temperatures higher than $\sim 1 \mathrm{keV}$.

These conclusions about the plasma temperature derived from the conditions of plasma overheating and of the shortage of

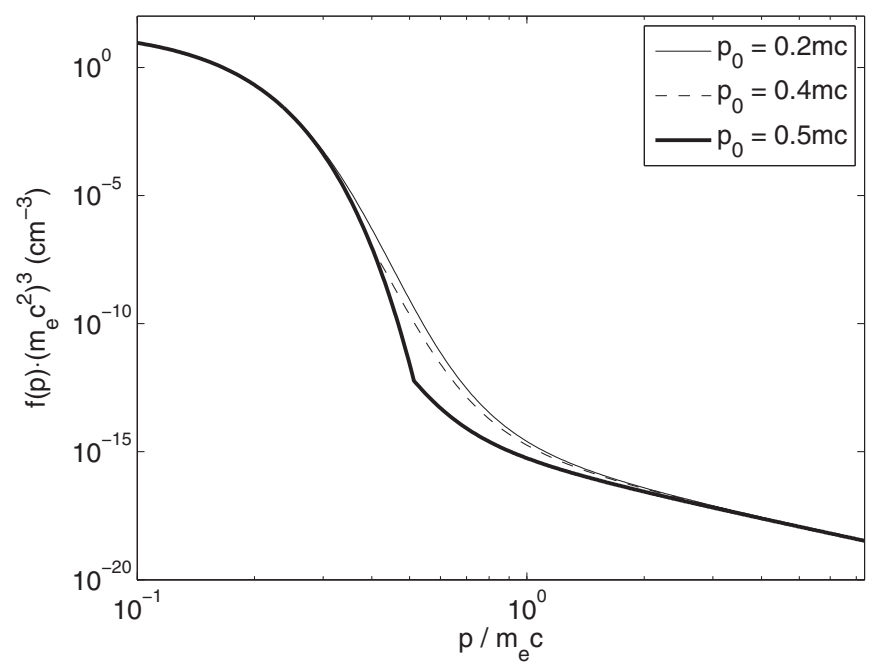

Figure 4. Spectrum of electrons in the bubbles for various values of $p_{0}$.

accelerated electrons are illustrated in Figure 3, where the plasma temperature, $T$, required for acceleration is shown as a function of the plasma density, $N$. The function $T(N)$ was derived for different thicknesses of the acceleration region $L$. The values along the curves satisfy the data $N=10^{-2} \mathrm{~cm}^{-3}$, $\mathrm{T}=2 \mathrm{keV}$ obtained by Su et al. (2010). We also placed the data obtained by Suzaku (see Kataoka et al. 2013) for the FB region in the figure. It seems to us that there is no serious discrepancy between results of numerical simulations and the observational data. The ROSAT data (see Snowden et al. 1997) for the FB region does not differ significantly from that of Suzaku.

These two effects of plasma overheating and of the shortage of accelerated electrons are illustrated in Figure 4, where we showed spectra of accelerated electrons for different $p_{0}$.

From this figure, one can see the reason for restricted values of $p_{0}$. For large values of $p_{0}$, the spectrum of electrons is below the thick solid line which showed the intensity of electrons needed for the observed gamma-ray flux. Just for this reason, we have a restriction that $\varsigma \lesssim 2.1$, shown by the vertical solid line in Figure 1. For $5>2$, the density of accelerated electrons is smaller than needed for the FB gamma-ray flux. On the other hand, the stochastic acceleration forms an excess 

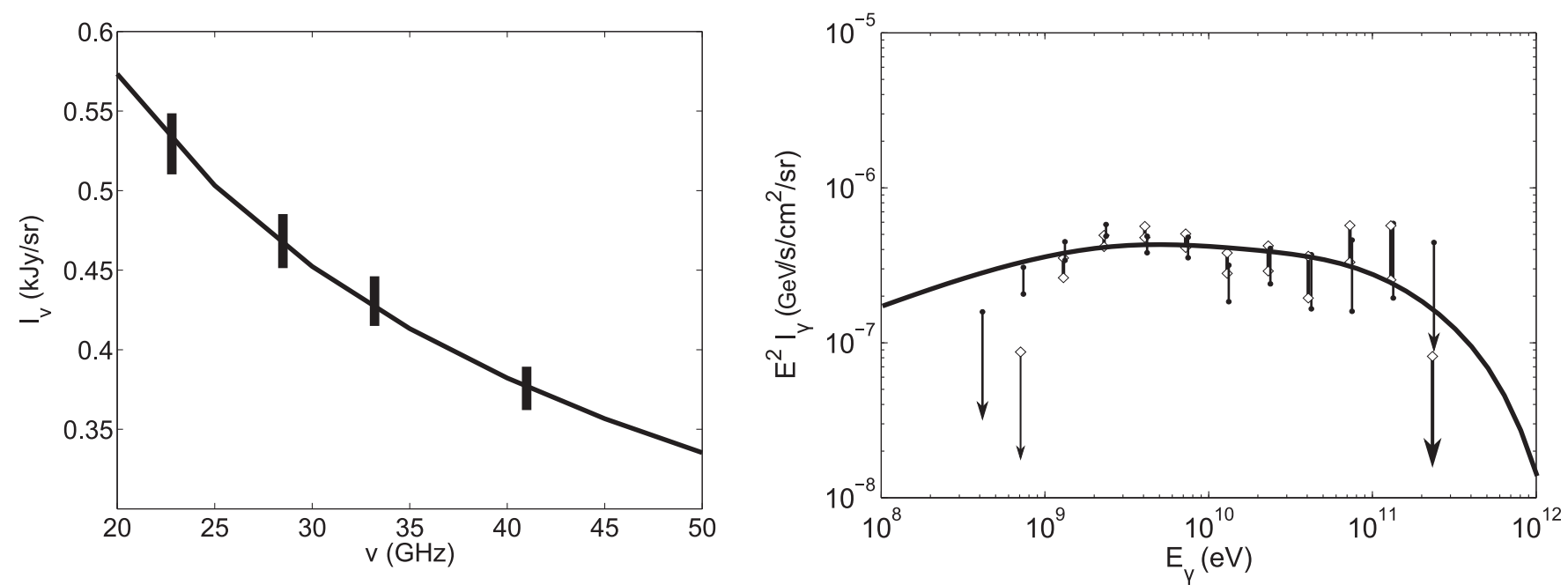

Figure 5. Spectrum of radio (left panel) and gamma-ray (right panel) emission from the FB. The data points were taken from Su et al. (2010) and Ade et al. (2013).

of suprathermal particles near the Maxwellian distribution. For $p_{0}<0.2$, this excess is so high that the electrons from this excess region effectively heat the plasma. Thus, the thermal pool absorbs the energy supplied by sources that prevents effective acceleration.

\section{NUMERICAL CALCULATIONS OF GAMMA-RAY AND RADIO EMISSION FROM THE FB}

Here, we present results of direct numerical calculations of the electron spectrum, gamma-ray, and radio emission when the distribution function $f$ is calculated numerically from Equation (1) for the derived parameters of acceleration. Then the FB gamma-ray spectrum is calculated from Equation (16). The expected radio spectrum at the frequency $v$ in the direction $\mathbf{I}$ is calculated from the following equation (see for details of the equation Syrovatskii 1959; Ginzburg \& Syrovatskii 1965):

$$
I_{r}(t, v, \mathbf{l})=\frac{1}{4 \pi} \int_{\mathbf{l}} d \mathbf{l} \int_{E} p(v, E) F(\mathbf{r}, E, t) d E,
$$

where $E=p c$ for relativistic electrons, $F(E)=$ $p^{2} f(p)(d p / d E)$, and the function $p(v, E)$ is

$$
p(v, E)=\sqrt{3} \frac{3 e^{3} H_{\perp}}{m c^{2}} \frac{v}{v_{c}} \int_{\nu / v_{c}}^{\infty} K_{5 / 3}(x) d x .
$$

Here, $H_{\perp}$ is the average component of magnetic field perpendicular to $\mathbf{l}$ and

$$
v_{c}=\frac{3 e H_{\perp}}{4 \pi m c}\left(\frac{E}{m c^{2}}\right)^{2} .
$$

For the derived values of $\varsigma, \alpha$, and $\tau$ (see the previous section), we numerically calculated the gamma-ray and radio intensity, which are shown in Figure 5. At that, the needed value of $p_{0}$ is $p_{0}=0.34$. The results of calculations coincide nicely with the data. Numerical calculations of the power of external sources as described by Equation (8) are shown in Figure 6. It is accepted here that the sources of acceleration are switched on at the time $t=0$. As one can see from the figure, the power reaches its stationary state at $\dot{W} \simeq 4.5 \times 10^{39} \mathrm{erg} \mathrm{s}^{-1}$ for the time $t=4 \times 10^{6} \mathrm{yr}$.

This value is lower than $10^{40} \mathrm{erg} \mathrm{s}^{-1}$, as estimated by Crocker \& Aharonian (2011) for the energy release provided by the star

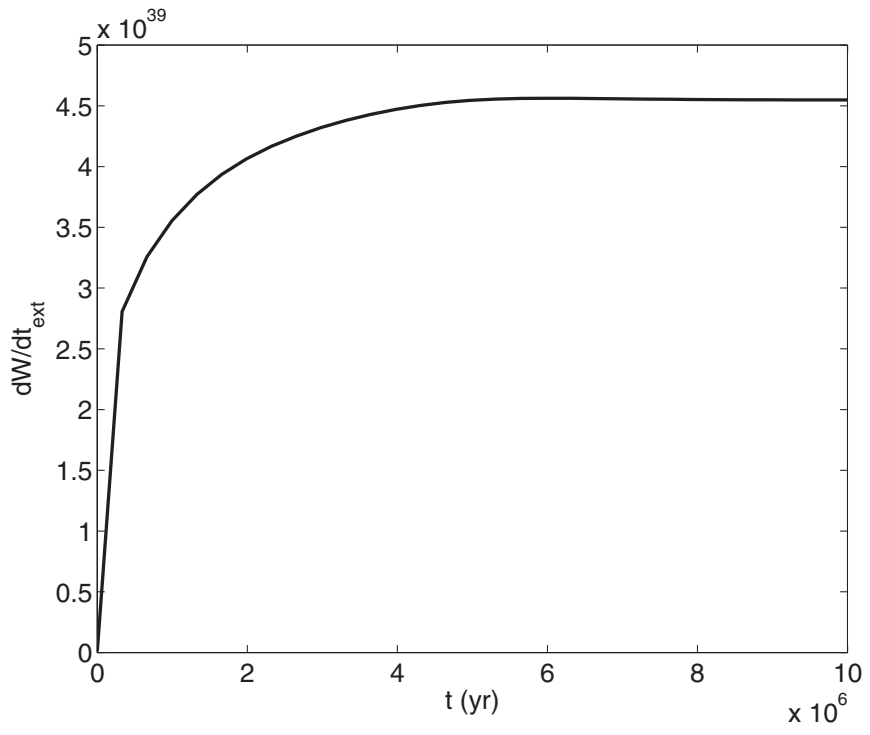

Figure 6. Evolution of the power supplied to the system with time.

formation regions in the $\mathrm{GC}$ and also is below $10^{41} \mathrm{erg} \mathrm{s}^{-1}$, as estimated by (Cheng et al. 2011) for star accretion processes onto the central black hole (condition 4). Thus, we conclude that the measured flux of radio and gamma-rays, the estimated power of sources, and the upper limit of energy release in the GC are in good agreement with each other in the model.

\section{CONCLUSION}

In order to provide high-energy electrons responsible for the electromagnetic radiation (gamma-ray and radio) from the Fermi bubbles, we investigated the case of stochastic in situ acceleration of electrons from the halo background plasma. The stochastic acceleration in the FB can be either due to charged particle interaction with resonant MHD waves or with a supersonic turbulence in the FB, as was assumed by Cheng et al. (2012). We obtained the following conclusions.

1. Two essential assumptions are used in the model. First, the FB gamma-ray emission is produced by the inverseCompton scattering of relativistic electrons on the background Galactic (IR and optical) and relic photons, and second, these electrons are accelerated by stochastic (Fermi) 
acceleration from the background Galactic plasma, whose density and temperature in the FB are accepted as $N=10^{-2}$ $\mathrm{cm}^{-3}$ and $T=2 \mathrm{keV}$. The process of stochastic acceleration in the FB can be either due to particle acceleration with a supersonic turbulence as assumed by Cheng et al. (2012) or by interactions with resonant MHD waves (see Berezinskii et al. 1990). The goal is to define model parameters at which the gamma-ray and radio emission from the FB can be provided by this acceleration mechanism.

2. As it is well known, the process of Fermi acceleration generates very flat (hard) spectra of particles that are harder than needed for the observed gamma-ray and radio emission from the FB. Besides, accelerated particles are accumulated nearby the $E_{\max }$ (see Equation (17)), forming there an excess of particles which also leads to a flat spectrum of the nonthermal emission generated by accelerated electrons. This pile-up effect is similar to that from the analysis of Vannoni et al. (2009) on electron acceleration by shocks. These problems of the model are eliminated by the term of particle escape with the time $\tau$. The escape term makes the spectrum steeper as needed for observations.

3. One of the main problems of stochastic acceleration from a background plasma is (over) heating of the plasma by accelerated particles, as shown by Wolfe \& Melia (2006); Petrosian \& East (2008), because the energy transferred to accelerated particles is quickly dumped into the thermal plasma. This effect prevents formation of nonthermal spectra. As Chernyshov et al. (2012) showed, however, the effect of overheating depends on parameters of acceleration, and it is insignificant if the stochastic acceleration is effective for particles with high enough momenta $p>p_{0}$. We determined parameters of acceleration when the acceleration of electrons in the FB is possible.

4. We described the stochastic (Fermi) acceleration as a momentum diffusion with the coefficient, $D_{F}(p)=$ $\alpha p^{\varsigma} \theta\left(p-p_{0}\right)$, where $p$ is the particle momentum, $p_{0}$ is a cutoff of the acceleration parameter, and $\alpha$ is the acceleration rate. The goal of our analysis is to define the model parameters $\alpha, \varsigma, p_{0}$, and $\tau$ at which the gamma-ray and radio emission from the FB can be provided by this acceleration mechanism.

5. The value of $p_{0}$ is determined from the conditions that the acceleration time $\tau_{\text {acc }} \sim 1 / \alpha$ is smaller that the time of the plasma heating by the acceleration particles $\tau_{T}$. We showed that for the case of bubble plasma, the effect of overheating is insignificant if the stochastic acceleration is effective for particles with a high cutoff momentum $p_{0} \simeq 0.34$, where $p_{0}$ is given in units of $m c$.

6. The required spectral index of the coefficient of momentum diffusion, $D_{F}(p)$, is $\varsigma=2$. The effect of particle escape from the acceleration region with the characteristic time $\tau$ is the steepening of the spectrum of acceleration particles. The spectrum required for the observed radio emission from the FB can be obtained if the escape time $\tau=1.1 \times 10^{13} \mathrm{~s}$ and the acceleration rate $\alpha \simeq 1.6 \times 10^{-14} \mathrm{~s}^{-1}$. As is clear from Equation (1), for $\varsigma=2$, the same effect of steepening can be obtained if particles lose their energy by adiabatic energy losses inside the FBs instead of escape from there. For the rate of adiabatic losses $d p / d t=-p / 3 \nabla \cdot \mathbf{u}$, the necessary spectral index of accelerated particles can be obtained if $1 / 3 \nabla \cdot \mathbf{u}=3 / \tau$.

7. As follows from our numerical calculations, the power supplied by external sources of acceleration in the FB
Table 1

Parameters of the Model of Stochastic Acceleration of Electrons in the FB

\begin{tabular}{ccccccc}
\hline \hline$T(\mathrm{keV})$ & $N\left(\mathrm{~cm}^{-3}\right)$ & $\mathrm{H}(\mu \mathrm{G})$ & $\varsigma$ & $\alpha\left(\mathrm{s}^{-1}\right)$ & $p_{0}$ & $\tau(\mathrm{s})$ \\
\hline 2 & 0.01 & 5 & 2 & $1.6 \times 10^{-14}$ & 0.34 & $1.1 \times 10^{13}$ \\
\hline
\end{tabular}

should be about $\sim 4 \times 10^{39} \mathrm{erg} \mathrm{s}^{-1}$. This is lower than $10^{40} \mathrm{erg} \mathrm{s}^{-1}$, as estimated by Crocker \& Aharonian (2011) for the energy release provided by the star formation regions in the $\mathrm{GC}$, and also is below $10^{41} \mathrm{erg} \mathrm{s}^{-1}$, as estimated by (Cheng et al. 2011) for star acceleration processes onto the central black hole.

8. In this model, the power excess between supplied by external sources and that emitted by electrons in the form of gamma-ray and radio fluxes can be removed from the FBs, either in the form particle escape from the bubbles or by particle interaction with the plasma outflow from the GC region (adiabatic losses).

9. In principle, a physical mechanism for a cutoff $p_{0}$ in the spectrum of MHD waves could be wave absorption by cosmic rays.

10. Our investigations showed that for chosen parameters of the background plasma in the $\mathrm{FB}$, the stochastic acceleration is able to provide the needed number of high-energy electrons in the FB if a set of the acceleration parameters is fixed. In Table 1, we summarized the required parameters of stochastic acceleration needed to reproduce the observed radio and gamma-ray emission from the FB.

The authors thank the unknown referee for careful reading of the text and many critical comments that helped to improve the paper. K.S.C. is supported by the GRF Grants of the Government of the Hong Kong SAR under HKU 701013. D.O.C. is supported in part by the LPI Educational-Scientific Complex, RFFI grant 12-02-31648, and Dynasty Foundation. D.O.C. and V.A.D. acknowledge support from RFFI grant 12-02-00005. C.M.K. is supported, in part, by the Taiwan National Science Council grant NSC 102-2112-M-008-019-MY3.

\section{APPENDIX \\ ANALYTICAL ESTIMATES OF THE CUTOFF MOMENTUM $\mathbf{p}_{0}$}

We present here a qualitative analysis as an illustration that MHD wave absorption by CRs may generate a cutoff in the spectrum of MHD waves. (Ptuskin et al. 2006, for the details of calculations and references, see). In the stationary case, the equation for spectral energy density of waves, $W(k, t)$, can be written as (see Norman \& Ferrara 1996)

$$
\frac{d \Pi(W, k, t)}{d k}=-2 \Gamma_{c r} W+\Phi \delta\left(k-k_{0}\right),
$$

where $k$ is the wavenumber and $\Phi$ is energy supplied by the external source at the scale $1 / k_{0} . \Gamma_{c r}$ is the term of wave absorption by CRs (see Berezinskii et al. (1990))

$$
\Gamma_{c r}(k)=\frac{\pi Z^{2} e^{2} V_{A}^{2}}{2 k c^{2}} \int_{p_{\mathrm{res}}(k)}^{\infty} \frac{d p}{p} F(p),
$$

where $p$ is the particle momentum, $p_{\text {res }}(k)=Z e B / c k$, $F(p)$ is the CR distribution. The number density of CRs is 
$N_{c r}=\int F(p) d p$ and $F(p)=p^{2} f(p)$, where $f(p)$ is the distribution function from Equation (1).

The term $d \Pi(W, k, t) d k$ describes the wave cascade. Spectrum of MHD turbulence in the interstellar medium is questionable, and, usually, Kraichnan or Kolmogoroff spectra are supposed for this medium. Below, we assume for simplicity that there is the Kraichnan spectrum in the FB; then, the equation for MHD waves can be presented in a compact form. For the Kraichnan spectrum, the wave cascade term is (see Ptuskin et al. 2006 and references therein)

$$
\left(\frac{d \Pi(W, k, t)}{d k}\right)_{K r}=\frac{d}{d k}\left[\frac{C\left(k^{3 / 2} W(k)\right)^{3 / 2}}{\rho V_{A}}\right] .
$$

Here, the constant $C \sim 1, V_{A}$ is the Alfven velocity, and $\rho$ is the plasma mass density.

The solution of Equation (A1) is given by

$$
\begin{aligned}
W(k)= & k^{-3 / 2}\left[k_{0}^{3 / 2} W\left(k_{0}\right)-\frac{Z^{2} e^{2} B^{2} V_{A}}{8 C c^{2}} \int_{k_{0}}^{k} d k_{1} k_{1}^{-5 / 2}\right. \\
& \left.\times \int_{p_{\text {res }}\left(k_{1}\right)}^{\infty} \frac{F(p) d p}{p}\right],
\end{aligned}
$$

where $W\left(k_{0}\right)=\sqrt{\rho V_{A} \Phi / C} k_{0}^{-3 / 2}$.

The coefficient of momentum diffusion $D_{p}$ is (see Berezinskii et al. 1990)

$$
D_{p}(p)=p^{2} \kappa(p),
$$

where

$$
\kappa(p)=\frac{12 \pi V_{A}^{2} k_{\mathrm{res}} W\left(k_{\mathrm{res}}\right)}{v r_{L} B^{2}} .
$$

Here, $B$ is the magnetic field strength, and $r_{L}$ is the particle Larmor radius $r_{L}=1 / k_{\text {res }}$.

From Equations (A4) and (A6), we have

$$
\kappa(p)=\kappa_{0}(p)\left(1-g \int_{p}^{p_{L}} x^{1 / 2} d x \int_{x}^{\infty} d y \frac{F(y)}{y}\right),
$$

where

$$
\kappa_{0}(p)=\frac{3 \pi}{4} \frac{V_{A}^{2} k_{L}^{3 / 2} W\left(k_{L}\right)}{v r_{L} B^{2}}
$$

is the diffusion coefficient determined by the Kraichnan spectrum; the coefficient $g$ is

$$
g=\sqrt{\frac{Z e}{\pi c \rho}} \frac{B^{3 / 2}}{16 C k_{0}^{3 / 2} W\left(k_{0}\right)} .
$$

From observations, the function $F(p)$ is supposed to be a power law with a spectral index between 1.8 and 2.4 (Su et al. 2010). To simplify the calculations, we take $F(p)$ in the form

$$
F(p)=\frac{8 \times 10^{-12}}{m c} p^{-2.25} \mathrm{~cm}^{-3} \mathrm{mc}^{-1}=\frac{\alpha_{p}}{m c} p^{-2.25} .
$$

The solution for $\kappa(p)$ can be obtained similar to that of Ptuskin et al. (2006). For the variable $x=p^{3 / 2}$ and the function $\phi=\left(\kappa(p) / \kappa_{0}(p)\right)$, we obtain from Equation (A7)

$$
\frac{d^{2} \phi}{d x^{2}}=-\frac{4 g \sqrt{m c} \alpha_{p}}{9} \frac{\phi(x)}{x^{2.5}} .
$$

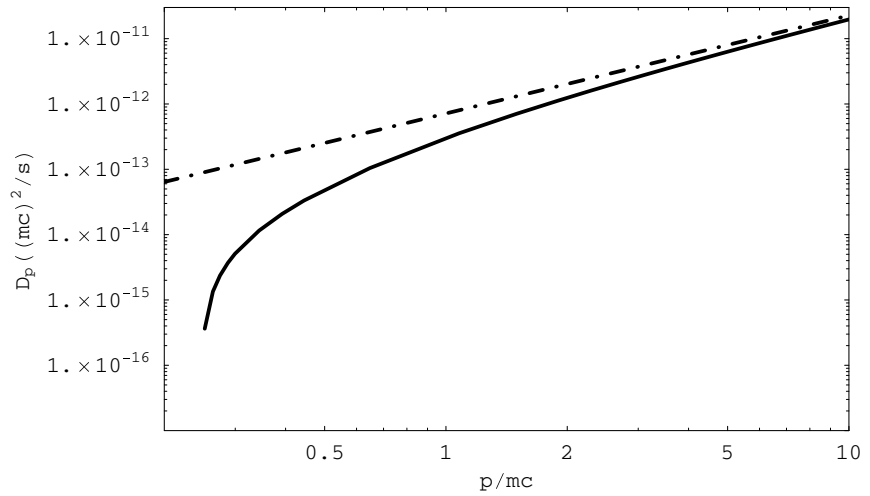

Figure 7. Solid line shows the momentum diffusion coefficient derived for the bubble parameters when the CR absorption is taken into account. The dash-dotted line is the result ignoring the CR absorption.

Solving Equation (A11) gives

$$
\kappa(p)=B \kappa_{0}(p) p^{3 / 2} J_{2}(\xi),
$$

where $B$ is a constant that can be defined from the boundary condition of $\kappa(p)=\kappa_{0}(p)$ at $p \rightarrow \infty$ :

$$
\xi(p)=\sqrt{\frac{64}{9} g \alpha_{p}}(m c)^{1 / 4} p^{-3 / 8} .
$$

At $\xi=5.14$, the Bessel function $J_{2}(\xi)=0$. This condition just determines the cutoff momentum in Equation (7).

If we take reasonable parameters for the $\mathrm{FB}$, the average energy release $\Phi=10^{39} \mathrm{erg} \mathrm{s}^{-1}$ and the plasma density $n=10^{-3} \mathrm{~cm}^{-3}$, we get $p_{0}=\simeq 0.2$.

The momentum diffusion coefficient $D_{p}$ for the bubble parameters is shown in Figure 7 (solid line). For comparison, the dash-dotted line is the diffusion coefficient for the Kraichnan spectrum of turbulence without CR absorption.

\section{REFERENCES}

Ackermann, M., Ajello, M., Atwood, W. B., et al. 2012, ApJ, 750, 3 Ade, P., Aghanim, N., Arnaud, M., et al. (Planck Collaboration) 2013, A\&A, 554,139

Aharonian, F., Akhperjanian, A. G., Anton, G., et al. 2009, ApJL, 695, 40 Berezinskii, V. S., Bulanov, S. V., Dogiel, V. A., Ginzburg, V. L., \& Ptuskin, V. S. 1990, in Astrophysics of Cosmic Rays, ed. V. L. Ginzburg (Amsterdam: North-Holland), Chapter IX

Bland-Hawthorn, J., \& Cohen, M. 2003, ApJ, 582, 246

Blumenthal, G. R., \& Gould, R. J. 1970, RvMP, 42, 237

Bykov, A. M., \& Toptygin, I. N. 1993, PhyU, 36, 1020

Carretti, E., Crocker, R. M., \& Staveley-Smith, L. 2013, Natur, 493, 66 Cheng, K.-S., Chernyshov, D. O., Dogiel, V. A., Ko, C.-M., \& Ip, W.-H. 2011, ApJL, 731, L17

Cheng, K.-S., Chernyshov, D. O., Dogiel, V. A., et al. 2012, ApJ, 746, 116

Chernyshov, D. O., Dogiel, V. A., \& Ko, C.-M. 2012, ApJ, 759, 113

Crocker, R. M., \& Aharonian, F. 2011, PRL, 106, 101102

Croston, J. H., Kraft, R. P., \& Hardcastle, M. J. 2007, ApJ, 660, 191

Dobler, G., \& Finkbeiner, D. P. 2008, ApJ, 680, 1222

Dobler, G., Finkbeiner, D. P., Cholis, I., et al. 2010, ApJ, 717, 825

Dobler, G. 2012, ApJL, 760, L8

Dogiel, V. A. 2000, A\&A, 357, 66

Dogiel, V. A., Colafrancesco, S., Ko, C. M., et al. 2007, A\&A, 461, 433

Feain, I. J., Cornwell, T. J., Ekers, R. D., et al. 2011, ApJ, 740, 17

Finkbeiner, D. P. 2004, ApJ, 614, 186

Ginzburg, V. L., \& Syrovatskii, S. I. 1965, ARA\&A, 3, 297

Guo, F., Metheus, W. G., Dobler, G., \& Oh, S. P. 2012, ApJ, 756, 182

Gurevich, A. V. 1960, Sov. Phys. JETP, 38, 1150

Hooper, D., \& Slatyer, T. R. 2013, PDU, 2, 118

Jones, D. I., Crocker, R. M., Reich, W., Ott, J., \& Aharonian, F. A. 2012, ApJL, 747, L12 
Junkes, N., Haynes, R. F., Harnett, J. L., \& Jauncey, D. L. 1993, A\&A, 269, 29

Kataoka, J., Tahara, M., Totani, T., et al. 2013, ApJ, 779, 57

Landau, L., \& Lifshitz, E. 1981, Physical Kinetics (Oxford: Pergamon Press)

Mertsch, P., \& Sarkar, S. 2011, PhRvL, 107, 1101

Mingo, B., Hardcastle, M. J., Croston, J. H., et al. 2011, ApJ, 731, 21

Mingo, B., Hardcastle, M. J., Croston, J. H., et al. 2012, ApJ, 758, 95

Norman, C. A., \& Ferrara, A. 1996, ApJ, 467, 280

Petrosian, V., \& East, W. E. 2008, ApJ, 682, 175

Ptuskin, V. S., Moskalenko, I. V., Jones, F. C., Strong, A. W., \& Zirakashvili, V. N. 2006, ApJ, 642, 902

Snowden, S. L., et al. 1997, ApJ, 485, 125
Stawarz, Ł., Tanaka, Y. T., Madejski, G., et al. 2013, ApJ, 766, 48

Strong, A. W., Porter, T. A., Digel, S. W., et al. 2010, ApJL, 722, L58

Su, M., Slatyer, T. R., \& Finkbeiner, D. P. 2010, ApJ, 724, 1044

Syrovatskii, S. I. 1959, SvA, 3, 22

Vannoni, G., Gabici, S., \& Aharonian, F. A. 2009, A\&A, 497, 17

Wolfe, B., \& Melia, F. 2006, ApJ, 638, 125

Yang, H.-Y. Karen, Ruszkowski, M., \& Zweibel, E. 2013, MNRAS, 436, 2432

Yang, R.-Z., Sahakyan, N., de Ona Wilhelmi, E., et al. 2012, A\&A, 542,19

Zubovas, K., \& Nayakshin, S. 2012, MNRAS, 424, 666 